evaluate the influence of the use of vaccine even without identification of who used the vaccine. The frequency of atypias was compared year by year. The research was approved by Ethical committee of Federal University of Ceará.

Results During nine years was performed 205056 Pap smears in the laboratory. In general the atypias (>ASC-US) occurred in 4927 cases $(2.4 \%)$. In 2006 the atypias (>ASC-US) occurred in 598 cases (2.24\%), and in 2007 in 739 cases (2.7\%). The vaccine was introduced in 2008 when the cytological atypia occurred in 873 cases (3.5\%). The in 2009, 2010, 2011, 2012, 2013 and 2014 the frequency was respectively, 598 (2.52\%), 456 (2.02\%), 540 (2.42\%), 437 (2.06\%), $349(1.72 \%)$ and $227(1.4 \%)$. When we evaluated only the women with less than 18 years old the results were: 2006416 (2.4); 2007 - 363 (3.6\%); 2008 - 389 (3.6\%); 2010 459 (2.6\%); 2011-440 (3.9\%); 2012 - 428 (1.9\%); 2013 $375(1.3 \%)$ and $2014-397$ (1,01\%).

Conclusion After the introduction of the HPV vaccines the cytological atypia in Pap smear decrease in Fortaleza - Brazil.

\section{P5.27 PREVALENCE OF CHLAMYDIA TRACHOMATIS IN DIFFERENT AGE GROUPS OF WOMEN IN FORTALEZA, BRAZIL}

Renata Mirian ${ }^{1}$ Nunes Eleuterio, ${ }^{1} J o s e ́$ Eleutério Junior, ${ }^{2}$ Natalice Lima, ${ }^{2}$ Maiara Nunes Alexandre. 'Universidade Federal do Ceará, Brazil; ${ }^{2}$ LABPEC, Brazil

\subsection{6/sextrans-2017-053264.643}

Introduction Chlamydia trachomatis infection is usually asymptomatic, affects young, sexually active women, and when not properly treated, can lead to complications such as pelvic inflammatory disease, infertility, and ectopic pregnancy.

Objectives To evaluate the prevalence of $C$. trachomatis in women classified into different age groups who presented at private clinics in the city of Fortaleza, Brazil.Methods: Data from samples of sexually active women who presented at general gynaecology clinics in the city of Fortaleza, Brazil, were processed using the Hybrid Capture method (Digene ) for the detection of DNA-Chlamydia trachomatis during 2014 and 2015.

Results A total of $11.22 \%$ and $8.24 \%$ of the women who presented at the clinic were positive for C. trachomatis in 2014 and 2015, respectively. In both years, the highest prevalence in different age groups was observed in women 40 years.

Conclusion During 2014 and 2015, the prevalence of Chlamydia trachomatis was high in the city of Fortaleza, especially in women.

\section{P5.28 DIAGNOSIS OF BACTERIAL VAGINOSIS WITH AFFIRM VPIII}

${ }^{1}$ Renata Mirian Nunes Eleuterio, ${ }^{1} J o s e ́$ Eleutério Junior, ${ }^{2}$ Paulo Giraldo, ${ }^{3}$ Ana Katherine Gonçalves, 'Beatriz Gordiano Valente, ${ }^{1}$ Fernanda Queiroz. ${ }^{1}$ Universidade Federal do Ceará, Brazil; ${ }^{2}$ Universidade Estadual de Campinas - UNICAMP, Brazil; ${ }^{3}$ Universidade Federal do Rio Grande do Norte, Brazil

\subsection{6/sextrans-2017-053264.644}

Introduction In gynaecology clinics, the most frequently reported women's complaint is associated with vaginitis and vaginosis. The three most common etiologies for vaginal symptoms are bacterial vaginosis (BV), vulvovaginal candidiasis, and trichomoniasis. Carefully recorded histories, examinations, and laboratory testing to determine the aetiology of vaginal symptoms are warranted.To assess the accuracy of Affirm VPIII in BV diagnosis in women with vaginal discharge.

Methods A cross-sectional study was performed in 73 women (mean age $32+9.7$ years) from March to November 2014 in a private gynaecology clinic in the Brazilian city of Fortaleza. All of the women compained of vaginal discharge. After speculum placement, we obtained material from the vaginal wall with a swab and stored it in tubes using the Ambient Temperature Transport System (ATTS). At the same time, another swab was used to collect material for the Gram test (gold standard). The project was approved by Ethical Committee of Federal University of Ceará.

Results The Affirm test identified Gardnerella vaginalis in 37/ 73 cases (50.7\%). Comparing the results from the Affirm test with Gram staining for BV indicated that the sensitivity for the Affirm test was $83.3 \%$, the specificity was $62.2 \%$, and the positive and negative predictive values were $20.8 \%$ and 97.1\%, respectively. The Kappa index between the tests was 0.204 , which was considered a fair degree of agreement.

Conclusion The Affirm test appears to be sensitive enough to diagnose BV with satisfactory specificity for G. vaginalis.

\section{P5.29 AWARENESS AND INTEREST IN PRE-EXPOSURE PROPHYLAXIS (PREP) AMONG YOUNG MEN ATTENDING A PUBLIC SEXUALLY TRANSMITTED DISEASES (STD) CLINIC IN A HIGH PREVALENCE URBAN SETTING}

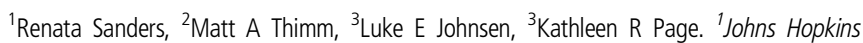
University, USA; ${ }^{2}$ Johns Hopkins School of Medicine, USA; ${ }^{3}$ Baltimore City Health Department/Johns Hopkins School of Medicine, USA

\subsection{6/sextrans-2017-053264.645}

Introduction Young men who have sex with men (YMSM), especially African American YMSM, are disproportionately affected by HIV. Pre-Exposure Prophylaxis (PrEP) is a highly effective biomedical intervention that could prevent new HIV infections among YMSM. We assessed awareness and interest in PrEP among young men receiving care at the Baltimore City Health Department (BCHD) Sexually Transmitted Diseases (STD) Clinics.

Methods We surveyed a convenience sample of 252 men ages 18-24 who attended BCHD clinics between 4/12/2016 and 10/3/2016. Participants were provided a self-administered survey that assessed awareness and interest in PrEP. Univariate and multivariable logistic regression analysis examined predictors of awareness and interest. Data collection continues to determine if awareness and interest change over time.

Results Mean age was $21.5(\mathrm{SD}=1.98)$ and the majority (93.7\%) self-identified as African American. Thirty-three participants (13.1\%) reported sex with other men. Among all participants, $19.4 \%$ were aware of PrEP, and $44.4 \%$ were interested in learning more about PrEP. Among YMSM, 72.7\% were aware of PrEP, 63.6\% were interested in learning more about PrEP, and 9.1\% reported current PrEP use. No current PrEP use was found in young men who did not report sex with men. In multivariable analysis adjusting for race, YMSM were more likely to be aware $(A O R=19.9$, $\mathrm{p}<0.001)$ and interested in $\operatorname{PrEP}(\mathrm{AOR}=2.5, \mathrm{p}=0.023)$ than young men who did not report sex with other men. Among YMSM, 37.5\% reported learning about PrEP from healthcare providers, $36.4 \%$ from friends or sexual contacts, and $24.2 \%$ from media (internet, television, phone apps). 
Conclusion YMSM receiving care at the BCHD STD Clinics are likely to be aware and interested in PrEP but fewer than $10 \%$ may be taking PrEP. Given that STD Clinics serve primarily minority young males who are disproportionately affected by HIV, future work should seek to improve youthfriendly services at public health clinics and leverage awareness and interest in PrEP in order to improve uptake in this vulnerable population.

\section{P5.30 ACCEPTABILITY OF PRE-EXPOSURE PROPHYLAXIS IN GAY MEN AND OTHER MSM IN GUATEMALA CITY}

Ricardo Mendizabal-Burastero, Jose Manuel Aguilar. Colectivo Amigos Contra El Sida, Guatemala

\subsection{6/sextrans-2017-053264.646}

Introduction Pre-exposure prophylaxis (PrEP) is one the most promising HIV interventions, with more than $90 \%$ efficacy with consistent adherence. In Guatemala, after more than 10 years of highly financed interventions, MSM continues to be one of the most affected populations for HIV epidemic. Other interventions, mostly behavioural, haven't changed the course of the HIV epidemic in these population. Our objective was to determinate the acceptability of PrEP in gay men and other MSM in Guatemala city.

Methods Exploratory qualitative study was performed between October and December 2015. 15 MSM were interviewed. Semi-structurated interview of 23 questions regarding: sociodemographic, risk scale, sexual practices and PrEP knowledge. Barriers and facilitators for PrEP were also asked. All participants signed an informed consent. Data analysis was perfomed on Atlas.Ti.

Results Median of age 32 years old, most of the participants reported higher education. Based on CDC guidelines for PrEP, 10 of the participants should be offered PrEP based on their current risks. Condom use was high, and for 3 participants that will be the preferred option always. 13 of them were likely to start PrEP if available, with fears about side effects and cost of the medications. Facilitators for PrEP consumtion was low cost, accessibility of the delivery point, counselling and knowledge about PrEP. To improve adherence, participants recommended phone alarms, text messages, extra pills, flexible or accessible hours for the service.

Conclusion PrEP has a high acceptability among MSM population in Guatemala city. Facilitators and barriers mentions will help to design a successful demonstration project. Based on the reported risks and HIV prevalence in this population, PrEP is an effective needed intervention in these country that can help to change the course of the epidemic. Based on these results, we have started a small fee-based demonstration project since 2016 .

\section{P5.31 SAFETY AND ACCEPTABILITY OF CELLULOSE SULFATEAS A VAGINAL MICROBICIDE IN HIV-INFECTED WOMEN}

Ronald Nyanzi Walakira. Mulago-Mbarara Teaching Hospital, Uganda

\subsection{6/sextrans-2017-053264.647}

Introduction Few studies of topical microbicides have assessed their safety in HIV-infected women. We conducted this study to evaluate the safety and acceptability of $6 \%$ cellulose sulfate
(CS) gel as a vaginal microbicide in sexually abstinent and active HIV-infected women.

Methods Fifty-nine HIV-infected women were enrolled in a randomised double-blind placebo-controlled study comparing $6 \%$ CS to placebo gel used for 14 days. Sexually abstinent women applied gel once or twice daily and sexually active women used gel once daily.

Results CS gel was safe with no reported severe or life-threatening adverse events (AF). Thirty-nine (66\%) of the participants experienced urogenital AE judged as probably or possibly related to gel. The majority $(51 \%)$ of these participants reported only mild events. Fewer women (62\%) who used CS experienced urogenital AE than those assigned to placebo gel $(70 \%) \quad(p=0.59)$. Eleven $(19 \%)$ women experienced intermenstrual bleeding judged to be probably or possibly related to gel use (four in the CS and seven in the placebo gel group). There was no increase in $\mathrm{AE}$ by frequency of gel use - sexual activity with the exception of abdominal/pelvic pain which was noted more frequently with twice daily use among sexually abstinent women. Women and men found the gel highly acceptable.

Conclusion This Phase I study demonstrated that CS vaginal gel was safe, well tolerated and acceptable by HIV-infected women and their male partners. Thus, further development of CS is warranted as a potential method to prevent HIV transmission and acquisition.

\section{P5.32 BACTERIAL VAGINOSIS AS A RISK FACTOR FOR ACQUIRING SEXUALLY TRANSMITTED DISEASES}

Ssempala Brian Adriane. Centre For Hiv,Std And Tb Prevention, Masaka, Uganda

\subsection{6/sextrans-2017-053264.648}

Introduction Few studies have demonstrated that Bacterial vaginosis (BV) is associated with sexual behaviour risk factors similar to those for other sexually transmitted diseases. In the present study, the prevalence of these in a multivariate analysis of data from sexually active women infected with BV and either Chlamydia trachomatis (CT), Treponema pallidum (syphilis), Neisseria gonorrhoeae (NG) or HIV was observed; NonBV infected women were used as control subjects.

Methods Data from 788 women screened in the SAVVY HIV gel phase III clinica! trial in Accra (West Legon Study Site) from 2012 to 2015 were analysed, Participants were evaluated for the presence of BV, CT, Treponema pallidum (PT), NG, Trichomonas vaginalis (TV) and Human Immunodeficiency Virus (HIV), and interviewed in detail with respect to sexual behaviours after consent forms were signed.

Results This study has shown a high association between BV and HIV $(\mathrm{p}<0.01)$ with risk factor $(0.4)$, which does not occurred in the other sexually transmitted diseases like NG, syphilis and Chlamydia with insignificant association $(\mathrm{p}<1)$ and risk factors $(0.6,0.7,0.9)$ respectively. HIV was found to be the most prevalent sexually transmitted disease with 11.2\%, Chlamydia 9.2\%, TV 2.3\%, Syphilis $1.7 \%$ and NG the least with $1.5 \%$.

Conclusion Bacteria associated with bacterial vaginosis increase female genital-tract infection of HIV but the mechanism by which this happens is not clear. Bacterial vaginosis is not a sexually transmitted disease but predisposes one to HIV infection. It is strongly suggested that all cases of BV both symptomatic and asymptomatic that are presented in the sexual- 